\title{
Epipelic cyanobacteria and algae: a case study from Czech ponds
}

\author{
Petr HaŠLeR ${ }^{1}$, Jana ŠTĚPÁnKovÁ ${ }^{1}$, Jana ŠPaČKovÁ ${ }^{1}$, Jiří Neustupa ${ }^{2}$, Miloslav \\ Kitner $^{1}$, Petr Hekera ${ }^{3}$, Jana Veselá ${ }^{2}$, Jaroslav Burian ${ }^{4} \&$ Aloisie PoulíčKovÁ ${ }^{1}$ \\ ${ }^{1}$ Department of Botany, Faculty of Science, Palacký University, Svobody 26, CZ-771 46 Olomouc, Czech \\ Republic; e-mail: petr.hasler@upol.cz, aloisie.poulickova@upol.cz \\ ${ }^{2}$ Department of Botany, Faculty of Science, Charles University, Benátská 2, CZ-128 01 Prague 2, Czech \\ Republic \\ ${ }^{3}$ Department of Ecology, Faculty of Science, Palacký University,Šlechtitelů 11, CZ-783 71 Olomouc, Czech \\ Republic \\ ${ }^{4}$ Department of Geoinformatics, Faculty of Science, Palacký University, Svobody 26, CZ-771 46 Olomouc, \\ Czech Republic
}

\begin{abstract}
The present paper focuses on the epipelic cyanobacteria and algae (particularly desmids). Altogether 45 sediment samples were taken at ponds covering a $\mathrm{pH} /$ conductivity and trophic gradients. Statistic evaluation based on environmental variables measured, divided localities into four major groups differing also by sediment quality and its algal flora. Altogether 39 cyanobacterial species were found including sedimented planktic or litoral forms, with prevalence of motile filamentous genera (Komvophoron, Oscillatoria, Phormidium, Pseudanabaena). Although, the majority of 42 desmid taxa belongs to commonly occurring species with a wide ecological amplitude, several remarkable taxa occurred mostly at oligo/dystrophic sites with sandy sediments. The highest species richness of euglenophytes was found on the muddy sediments (both oxygenated and anoxic). Other epipelic organisms were represented by Gymnodinium aeruginosum, Paulinella chromatophora and various protozoa, feeding on epipelic algae (Amoeba, Urceolus cyclostomus).
\end{abstract}

Key words: epipelon, cyanobacteria, desmids, ecology

\section{Introduction}

Epipelic algae can perform a range of ecosystem functions, that include biostabilisation of sediments, regulation of benthic-pelagic nutrient cycling, and primary production. There is a growing need to understand their ecological role in light of current and future alterations in sediment loading resulting from land-use change and land management practices (PoulíčKovÁ et al. 2008a). The study of epipelic (cyanobacteria and eukaryotic algae that live on or in association with finegrained substrata) algal ecology was pioneered within freshwater habitats by Round $(1953,1957$, 1961, 1972). However, interest did not develop to the same extent as in other important areas of freshwater research, most prominently the study of eutrophication and phytoplankton ecology.

Lake/pond sediments differ in structure, chemical composition and in inhabiting organisms. Freshwater epipelic assemblages are mainly dominated by diatoms, cyanobacteria, euglenopytes, cryptophytes, dinophytes and chlorophytes, particularly by motile forms (LysÁKovÁ et al. 2007, PoulíčKovÁ et al. 2008a).

Motility seems to be a common feature of most autochthonous epipelic cyanobacteria and algae allowing them to migrate vertically within sediments (Round \& EATON 1966, HAPPEYWood 1988). In addition, resting stages and settled cells of planktic algae (allochtonous part of bentic assemblages) can be found on the bottom (Sicko-GoAd et al. 1989, Belmonte et al. 1997). The bottom sediment is an important source of nutrients, their cycles strongly depend on microorganisms inhabiting bottom anaerobic/ aerobic microhabitats (LOCK et al. 1984, PAERL 1990).

The microorganisms distribution on the lake/pond bottom is influenced by environmental variables, particularly temperature, oxygen, light, chemical gradients (BURKHOLDER 1996, PoulíčKová et al. 2008a).

Although epipelic diatoms represent a model 
assemblage for studies on reproductive biology, cryptic speciation and geographic biodiversity (Mann \& Droop 1996, Mann 1999, Mann et al. 1999, LysákOvá et al. 2007, MANn et al. 2008, PoulíčKová et al. 2008b), except for the studies by Round $(1959,1961,1972)$ epipelic cyanobacteria and algae have been largely overlooked.

The present study focuses on diversity of epipelic cyanobacterial and algal (particularly desmid) flora of the Czech Republic in relation to selected environmental variables.

\section{Methods}

Altogether 45 sediment samples were taken in May 2007 at sites (Fig. 1., Table 1), covering a pH/ conductivity and trophic gradients (from dystrophic/ oligo-mesotrophic to alkalic, eutrophic/hypertrophic ponds).

Samples were taken using a glass tube as described by Round (1953). The mud-water samples were poured out into plastic boxes and allowed to stand in the dark for at least $5 \mathrm{~h}$. Then the supernatant was removed by suction and the mud covered with lens tissue. In response to the continuous illumination provided, epipelic algae moved up through the lens tissue and attached themselves to cover slips placed on top. These were removed at intervals and either examined immediately, or used for isolation (by streaking) on agar plates, or incubated in (1) Zehnder medium (Staub 1961) and (2) Bristol-Bold medium (BoLd 1949). Incubated cover slips and cultures were maintained at $18^{\circ} \mathrm{C}$ under cool-white fluorescent lights (irradiation of $20 \mu \mathrm{mol} . \mathrm{m}^{-2} \cdot \mathrm{s}^{-1}$ ) with $12 \mathrm{~h}$ light per day.

Environmental variables (temperature, $\mathrm{pH}$, conductivity) were measured in situ using instruments from the WTW company (Wissenschaftlich-Technische Werkstätten GmbH, Weilheim, Germany), transparency was measured in situ using a Secchi disc. Nutrient and chlorophyll-a concentration were analysed following standard methods (VERNON 1960, HEKERA 1999).

Photomicrography was carried out using a Zeiss Axioimager with a Zeiss Axiocam HRc digital camera (images captured and managed via Imaging Associates/ Zeiss Axiovision Version 4.5 imaging software). Bright field $(\mathrm{BF})$ or differential interference contrast (DIC) optics were used at $\times 100$ (planapochromat lenses, nominal numerical aperture 1.32 or 1.4 ).

Hierarchical clustering analysis was carried out with the environmental variables (Ward's method, NCSS software).

\section{Results}

The ecological evaluation of investigated ponds

Statistic evaluation based on environmental

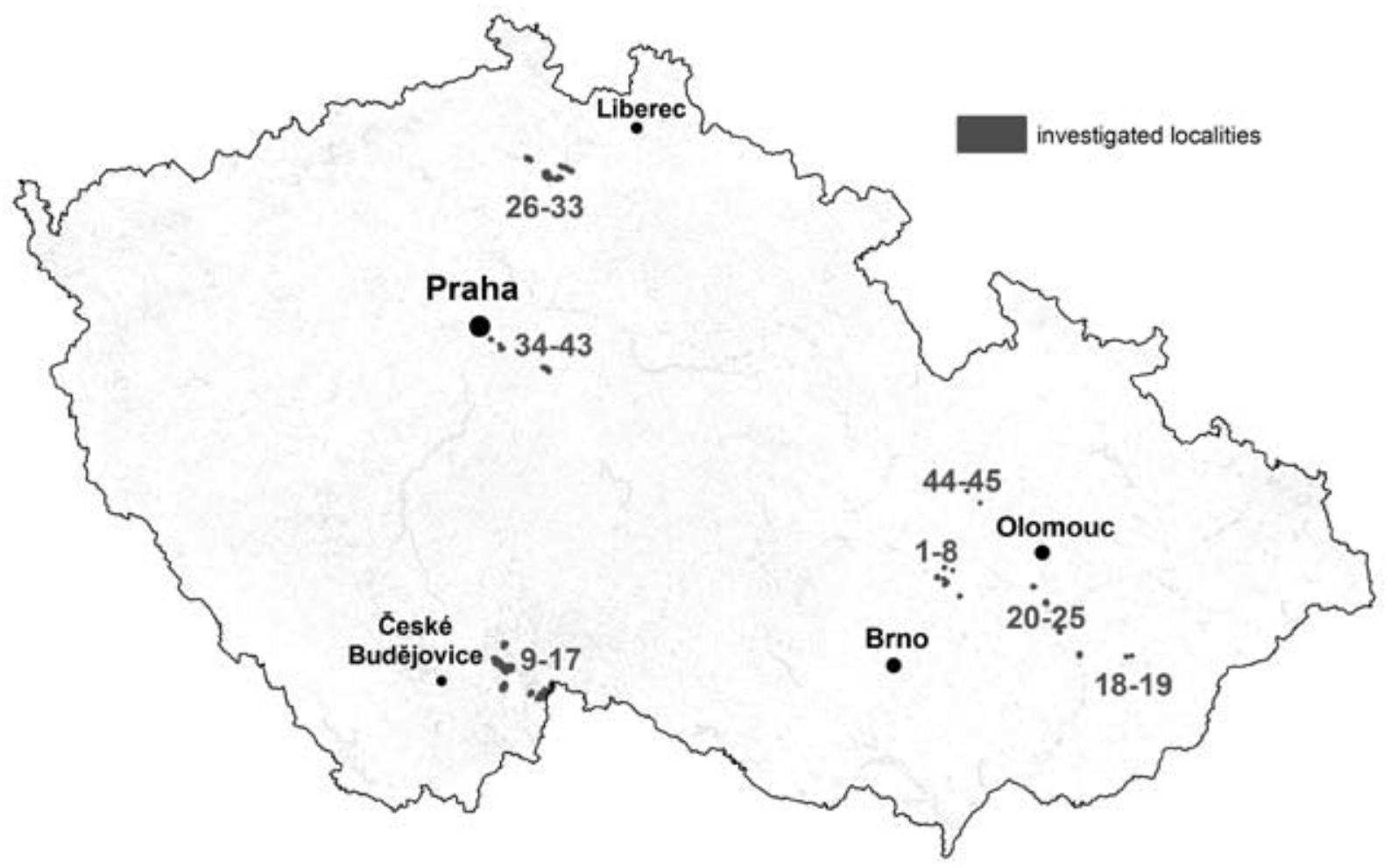

Fig. 1. Map of investigated localities in the Czech Republic. For detail description see Table 1. 
variables measured (Table 1), divided localities into four major groups (Fig. 2). Group no. I. includes ponds with low conductivity (below $300 \mu \mathrm{S} . \mathrm{cm}^{-1}$ ), high $\mathrm{pH}$ (usually above 9) and the highest concentration of total nitrogen from all samples. Bottom sediments of these localities (Buková, Drahany, Protivanov) can be characterized by medium-high proportion of sand grains (sandy-muddy). Group no. II. includes sites (e.g. Hamerský, Louňovický, Vrah, Hrdibořice, Tovačov, Záhlinice) characterized by high conductivity (usually above $500 \mu \mathrm{S} . \mathrm{cm}^{-1}$ ), slightly alkalic $\mathrm{pH}$ (7.5-8.5) and low nitrogen concentration (usually 2-2.5 mg. $\mathrm{l}^{-1}$ ). The sediments contained a black surface layer of detritus and organic material. Group no. III. includes oligo/dystrophic ponds (Břehyňský, Strážovský, Pavlov, U třech krátkých) with low conductivity and $\mathrm{pH}$ (see Table 1). The collected sediments were usually sandy with a very low portion of decomposing organic material. Group no. IV. mostly included eutrophic ponds with muddy or muddy-sandy sediments (Rožmberk, Starý kanclír, Velký Tisý, Bezedník) with very low conductivity (below $200 \mu \mathrm{S} . \mathrm{cm}^{-1}$ ), alkalic $\mathrm{pH}$ (above 8 ) and very low N/P ratio.

\section{Occurrence of cyanobacteria}

Cyanobacteria formed an important part within the epipelon. Altogether 39 species were found including sedimented planktonic or littoral species (Table 2, Figs 3-16), with prevalence of motile filamentous forms. We noticed differences in species richness and abundance of cyanobacteria among sampling sites, the highest being at sites with sandy-muddy sediments (groups No. IV., pond Bezedník, Horní Ves), in contrast to uniform substrate (only sandy/muddy; group No. I., pond Protivanov). Sites with anoxic muddy sediments (group No. II., pond Vrah) were poorly colonised.

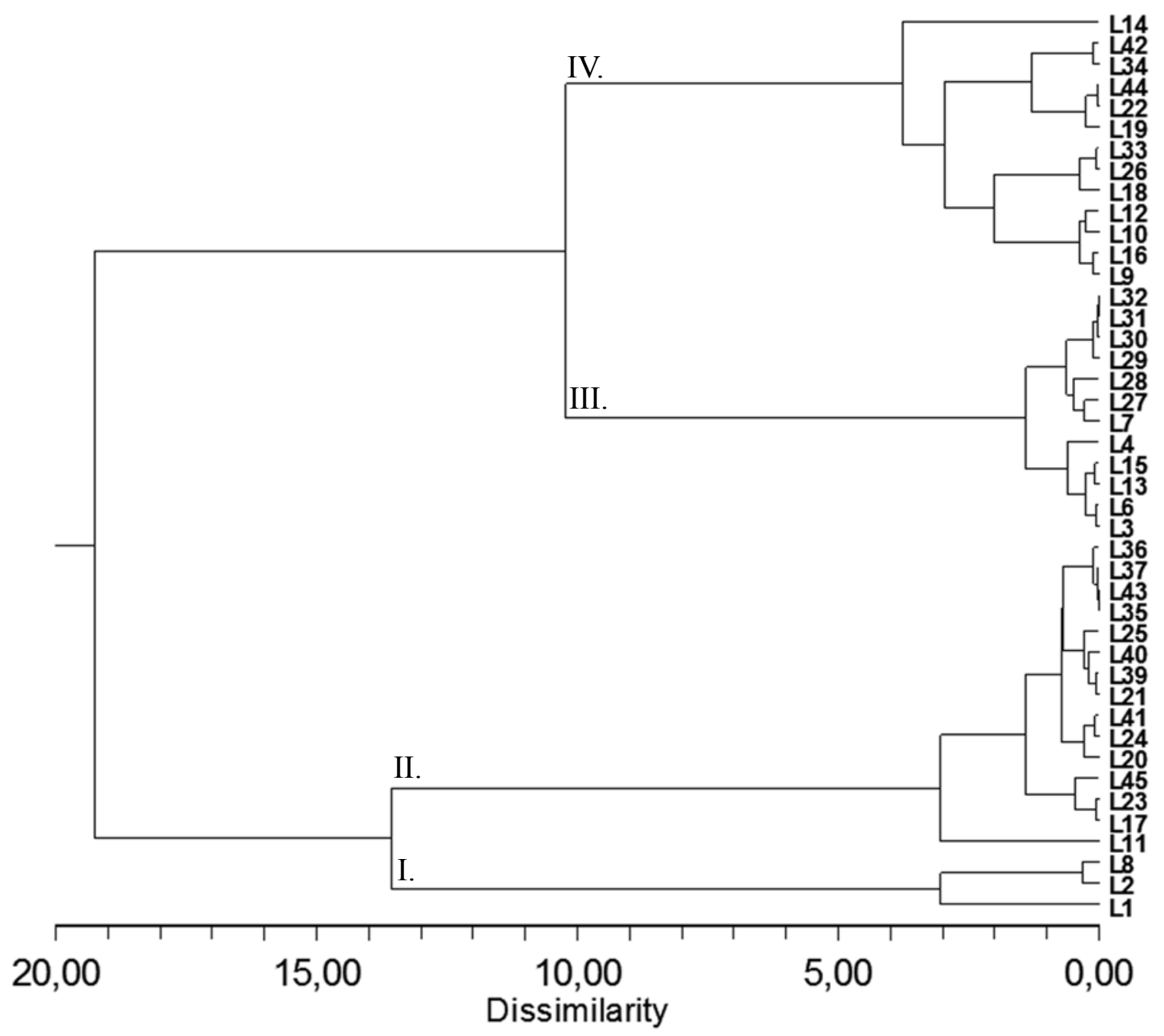

Fig. 2. Hierarchical clustering analysis of the investigated localities, based on the environmental variables (Ward's method). 
Table 1. Measured environmental variables (Alt - altitude in m.a.s.l., Shad - shading by surrounding vegetation in \%, Temp temperature in ${ }^{\circ} \mathrm{C}$, Cond - conductivity in $\mu \mathrm{S} . \mathrm{cm}^{-1}$, Trans - transparency in $\mathrm{cm}, \mathrm{N}_{\text {tot }}$ - total nitrogen in mg. $\mathrm{l}^{-1}, \mathrm{P}_{\text {tot }}-$ total phosporus in mg. $1^{-1}$, Alk - alkalinity in $\mu \mathrm{mol}, \mathrm{Si}$ - silica in mg. $1^{-1}$, Chl- $a$ - chlorophyl a concentration in $\mu \mathrm{g} \cdot \mathrm{l}^{-1}$ ).

\begin{tabular}{|c|c|c|c|c|c|c|c|c|c|c|c|c|c|}
\hline No. & Locality & GPS & $\stackrel{ \pm}{2}$ & 胥 & 芵 & రై & $\frac{\pi}{2}$ & 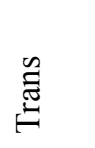 & $z^{\stackrel{\Xi}{\sigma}}$ & $\rho^{\breve{a}}$ & 光 & $\bar{\omega}$ & $\frac{i}{0}$ \\
\hline L1 & Drahany & $\begin{array}{l}\text { N } 49 ; 25 ; 54 ; 8 \\
\text { E } 16 ; 52 ; 34 ; 9\end{array}$ & 380 & 0 & 14.1 & 195 & 7.38 & 120 & 6.5 & 0.13 & 0.29 & 3.488 & 16.12 \\
\hline L2 & Protivanov & $\begin{array}{l}\text { N } 49 ; 28 ; 12 ; 2 \\
\text { E } 16 ; 48 ; 41 ; 7\end{array}$ & 615 & 0 & 13.4 & 201 & 9.93 & 240 & 6.5 & 0.16 & 0.29 & 3.085 & 6.38 \\
\hline L3 & Obora & $\begin{array}{l}\text { N } 49 ; 27 ; 44 ; 3 \\
\text { E } 16 ; 47 ; 54 ; 9\end{array}$ & 610 & 10 & 14.0 & 175 & 7.50 & 270 & 0.8 & 0.12 & 0.27 & 4.907 & 3.78 \\
\hline L4 & U 3 krátkých & $\begin{array}{l}\text { N } 49 ; 28 ; 47 ; 5 \\
\text { E } 16 ; 47 ; 35 ; 0\end{array}$ & 610 & 50 & 11.7 & 112 & 6.76 & 220 & 0.3 & 0.21 & 0.20 & 6.616 & 5.53 \\
\hline L5 & Suchý 1 & $\begin{array}{l}\text { N } 49 ; 28 ; 52 ; 5 \\
\text { E } 16 ; 45 ; 49 ; 5\end{array}$ & 673 & 0 & 14.9 & 290 & 10.90 & 40 & 5.9 & 0.62 & 0.98 & 2.409 & 265.47 \\
\hline L6 & Suchý 2 & $\begin{array}{l}\text { N } 49 ; 28 ; 54 ; 5 \\
\text { E } 16 ; 45 ; 40 ; 2\end{array}$ & 674 & 0 & 15.0 & 125 & 7.82 & 130 & 0.9 & 0.09 & 0.13 & 2.042 & 6.17 \\
\hline L7 & Pavlov & $\begin{array}{l}\text { N } 49 ; 30 ; 57 ; 7 \\
\text { E } 16 ; 47 ; 23 ; 6\end{array}$ & 680 & 30 & 14.7 & 200 & 7.28 & 140 & 1.9 & 0.06 & 0.51 & 2.986 & 9.40 \\
\hline L8 & Buková & $\begin{array}{l}\text { N 49;30;39;4 } \\
\text { E16;49;51;4 }\end{array}$ & 630 & 0 & 16.7 & 181 & 9.41 & 50 & 5.1 & 0.16 & 0.47 & 1.680 & 32.84 \\
\hline L9 & Naděje & $\begin{array}{l}\text { N } 49 ; 07 ; 07 ; 7 \\
\text { E } 14 ; 44 ; 31 ; 3\end{array}$ & 430 & 80 & 16.8 & 220 & 8.89 & 75 & 0.7 & 0.24 & 5.68 & 0.888 & 10.68 \\
\hline L10 & Velký Tisý & $\begin{array}{l}\text { N } 49 ; 04 ; 04 ; 2 \\
\text { E } 14 ; 42 ; 25 ; 6\end{array}$ & 429 & 0 & 15.3 & 245 & 8.33 & 190 & 0.5 & 0.23 & 3.00 & 1.730 & 45.01 \\
\hline L11 & Malý Tisý & $\begin{array}{l}\text { N } 49 ; 03 ; 13 ; 8 \\
\text { E } 14 ; 44 ; 57 ; 0\end{array}$ & 435 & 10 & 16.8 & 245 & 8.18 & 35 & 3.0 & 0.69 & 4.00 & 1.333 & 70.40 \\
\hline L12 & Rožmberk & $\begin{array}{l}\text { N } 49 ; 02 ; 53 ; 3 \\
\text { E } 14 ; 45 ; 43 ; 6\end{array}$ & 441 & 20 & 17.5 & 205 & 8.59 & 85 & 1.9 & 0.28 & 2.10 & 2.880 & 29.95 \\
\hline L13 & Opatovický & $\begin{array}{l}\text { N } 48 ; 59 ; 13 ; 9 \\
\text { E } 14 ; 46 ; 43 ; 4\end{array}$ & 445 & 50 & 17.4 & 215 & 7.60 & 55 & 1.1 & 0.24 & 2.62 & 0.922 & 34.06 \\
\hline L14 & Starý kanclír & $\begin{array}{l}\text { N } 48 ; 58 ; 05 ; 6 \\
\text { E } 14 ; 53 ; 43 ; 6\end{array}$ & 455 & 40 & 18.9 & 165 & 10.38 & 35 & 1.8 & 0.39 & 2.52 & 0.532 & 140.49 \\
\hline L15 & Hejtman & $\begin{array}{l}\text { N } 48 ; 57 ; 32 ; 4 \\
\text { E } 14 ; 56 ; 20 ; 8\end{array}$ & 469 & 0 & 23.4 & 132 & 7.65 & 110 & 0.4 & 0.19 & 0.85 & 0.376 & 19.26 \\
\hline L16 & Staňkov & $\begin{array}{l}\text { N } 48 ; 58 ; 31 ; 9 \\
\text { E } 14 ; 57 ; 26 ; 7\end{array}$ & 483 & 0 & 19.5 & 133 & 9.16 & 80 & 1.0 & 0.15 & 1.02 & 0.964 & 25.79 \\
\hline L17 & Špačkov & $\begin{array}{l}\text { N } 48 ; 58 ; 31 ; 9 \\
\text { E } 14 ; 57 ; 26 ; 7\end{array}$ & 483 & 10 & 18.2 & 188 & 7.76 & 25 & 2.4 & 0.39 & 3.33 & 2.127 & 66.19 \\
\hline L18 & Bezedník & $\begin{array}{l}\text { N } 49 ; 17 ; 58 ; 2 \\
\text { E } 17 ; 43 ; 35 ; 1\end{array}$ & 323 & 50 & 13.9 & 461 & 9.10 & 200 & 0.1 & 0.13 & 4.13 & 1.906 & 4.05 \\
\hline L19 & Horní Ves & $\begin{array}{l}\text { N } 49 ; 17 ; 45 ; 0 \\
\text { E } 17 ; 42 ; 03 ; 7\end{array}$ & 316 & 10 & 15.3 & 429 & 8.10 & 120 & 0.9 & 0.16 & 8.87 & 3.168 & 9.34 \\
\hline L20 & Záhlinice 1 & $\begin{array}{l}\text { N } 49 ; 17 ; 14 ; 6 \\
\text { E } 17 ; 28 ; 41 ; 1\end{array}$ & 198 & 0 & 15.7 & 670 & 7.93 & 30 & 3.3 & 0.38 & 2.88 & 2.982 & 69.00 \\
\hline L21 & Záhlinice 2 & $\begin{array}{l}\text { N } 49 ; 17 ; 14 ; 6 \\
\text { E } 17 ; 28 ; 41 ; 1\end{array}$ & 198 & 10 & 16.0 & 770 & 7.78 & 40 & 1.7 & 0.35 & 3.54 & 2.525 & 88.13 \\
\hline L22 & Chropyně & $\begin{array}{l}\text { N } 49 ; 21 ; 25 ; 4 \\
\text { E } 17 ; 22 ; 14 ; 1\end{array}$ & 207 & 50 & 15.5 & 422 & 7.68 & 80 & 1.5 & 0.24 & 2.44 & 1.932 & 28.02 \\
\hline
\end{tabular}


Table 1 Cont.

\begin{tabular}{|c|c|c|c|c|c|c|c|c|c|c|c|c|c|}
\hline \multirow[t]{2}{*}{ No. } & \multirow[t]{2}{*}{ Locality } & \multirow[t]{2}{*}{ GPS } & \multirow[b]{2}{*}{$\stackrel{ \pm}{<}$} & \multirow[b]{2}{*}{$\begin{array}{l}\vec{\Xi} \\
\text { ज }\end{array}$} & \multirow[b]{2}{*}{ 宅 } & \multirow[b]{2}{*}{ ర్̃ } & \multirow[b]{2}{*}{$\frac{\pi}{2}$} & \multirow[b]{2}{*}{$\stackrel{\text { 气 }}{\tilde{E}}$} & \multirow[b]{2}{*}{$z^{\underline{m}}$} & \multirow[b]{2}{*}{ 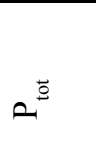 } & \multirow[b]{2}{*}{$\ddot{z}$} & \multirow[b]{2}{*}{$\bar{s}$} & \multirow[b]{2}{*}{$\frac{i}{d}$} \\
\hline & & & & & & & & & & & & & \\
\hline $\mathrm{L} 23$ & Tovačov & $\begin{array}{l}\text { N 49;26;06;8 } \\
\text { E 17;17;35;6 }\end{array}$ & 206 & 50 & 15.9 & 267 & 7.38 & 25 & 2.3 & 0.39 & 1.29 & 3.510 & 97.52 \\
\hline L24 & Hrdibořice 1 & $\begin{array}{l}\text { N } 49 ; 28 ; 56 ; 1 \\
\text { E } 17 ; 13 ; 31 ; 2\end{array}$ & 213 & 50 & 16.1 & 726 & 7.94 & 40 & 1.9 & 0.44 & 5.37 & 2.467 & 64.38 \\
\hline $\mathrm{L} 25$ & Hrdibořice 2 & $\begin{array}{l}\text { N } 49 ; 28 ; 56 ; 1 \\
\text { E } 17 ; 13 ; 31 ; 2\end{array}$ & 213 & 10 & 17.5 & 729 & 8.00 & 40 & 1.5 & 0.20 & 5.01 & 2.276 & 37.48 \\
\hline L26 & $\begin{array}{l}\text { Máchovo } \\
\text { lake }\end{array}$ & $\begin{array}{l}\text { N } 503434,4 \\
\text { E } 14 ; 39 ; 00 ; 0\end{array}$ & 266 & 0 & 15.9 & 303 & 8.70 & 40 & 1.3 & 0.02 & 1.96 & 1.599 & 11.20 \\
\hline L27 & Břehyňský & $\begin{array}{l}\text { N } 50 ; 34 ; 32 ; 9 \\
\text { E } 14 ; 41 ; 35 ; 7\end{array}$ & 266 & 30 & 17.6 & 203 & 7.09 & 70 & 0.4 & 0.02 & 1.21 & 1.259 & 10.60 \\
\hline L28 & Černý & $\begin{array}{l}\text { N } 50 ; 36 ; 30 ; 6 \\
\text { E } 14 ; 45 ; 46 ; 1\end{array}$ & 279 & 30 & 11.1 & 306 & 7.69 & - & 0.9 & 0.02 & 2.48 & 3.570 & 2.30 \\
\hline L29 & Vavrouškův & $\begin{array}{l}\text { N } 50 ; 36 ; 35 ; 4 \\
\text { E } 14 ; 45 ; 01 ; 9\end{array}$ & 287 & 10 & 18.1 & 293 & 8.05 & 240 & 0.3 & 0.02 & 2.20 & 2.494 & 8.76 \\
\hline L30 & Strážovský & $\begin{array}{l}\text { N } 50 ; 36 ; 38 ; 3 \\
\text { E } 14 ; 44 ; 29 ; 8\end{array}$ & 279 & 10 & 18.3 & 299 & 7.77 & 250 & 0.1 & 0.01 & 2.07 & 2.866 & 6.31 \\
\hline L31 & Tůn̆ u letiště & $\begin{array}{l}\text { N } 50 ; 36 ; 51 ; 0 \\
\text { E } 14 ; 43 ; 48 ; 1\end{array}$ & 289 & 50 & 19.0 & 221 & 7.50 & 150 & 0.1 & 0.03 & 1.13 & 1.568 & 6.73 \\
\hline L32 & Hradčanský & $\begin{array}{l}\text { N } 50 ; 37 ; 05 ; 6 \\
\text { E } 14 ; 42 ; 26 ; 5\end{array}$ & 287 & 30 & 18.4 & 245 & 7.57 & 120 & 0.1 & 0.01 & 1.68 & 2.009 & 13.46 \\
\hline L33 & $\begin{array}{l}\text { Novozámec- } \\
\text { ký }\end{array}$ & $\begin{array}{l}\text { N } 50 ; 37 ; 44 ; 7 \\
\text { E } 14 ; 32 ; 12 ; 1\end{array}$ & 261 & 0 & 19.7 & 332 & 8.90 & 140 & 0.8 & 0.01 & 2.05 & 3.700 & 7.68 \\
\hline L34 & Hostivař & $\begin{array}{l}\text { N } 50 ; 02 ; 23 ; 3 \\
\text { E } 14 ; 31 ; 53 ; 6\end{array}$ & 262 & 30 & 19.1 & 437 & 8.46 & 55 & 1.8 & 0.31 & 1.34 & 1.998 & 26.08 \\
\hline L35 & Hamerský & $\begin{array}{l}\text { N } 50 ; 03 ; 08 ; 3 \\
\text { E } 14 ; 29 ; 16 ; 7\end{array}$ & 220 & 50 & 18.6 & 748 & 7.47 & 30 & 2.6 & 0.34 & 1.74 & 3.504 & 48.32 \\
\hline L36 & Vrah1 & $\begin{array}{l}\text { N } 50 ; 01 ; 50 ; 9 \\
\text { E } 14 ; 32 ; 53 ; 4\end{array}$ & 263 & 30 & 19.3 & 722 & 7.17 & 45 & 2.5 & 0.41 & 1.69 & 5.439 & 42.57 \\
\hline L37 & Vrah2 & $\begin{array}{l}\text { N } 50 ; 01 \\
\text { E } 14 ; 32\end{array}$ & 274 & 30 & 19.6 & 543 & 7.32 & 30 & 2.2 & 0.35 & 2.37 & 2.768 & 33.25 \\
\hline L38 & Homolka & $\begin{array}{l}\text { N } 50 ; 01 ; 38 ; 4 \\
\text { E } 14 ; 32 ; 42 ; 1\end{array}$ & 212 & 70 & 17.3 & 660 & 9.82 & 50 & 3.4 & 0.49 & 1.40 & 9.549 & 6.73 \\
\hline L39 & Milíčov & $\begin{array}{l}\text { N } 50 ; 01 ; 34 ; 0 \\
\text { E } 14 ; 32 ; 27 ; 0\end{array}$ & 302 & 30 & 19.5 & 778 & 7.65 & 50 & 2.0 & 0.28 & 3.08 & 2.710 & 19.13 \\
\hline L40 & Požár & $\begin{array}{l}\text { N 49;59;15;5 } \\
\text { E14;45;24;2 }\end{array}$ & 419 & 0 & 19.3 & 556 & 7.59 & 55 & 2.9 & 0.26 & 2.15 & 4.526 & 5.06 \\
\hline L41 & Louňovický & $\begin{array}{l}\text { N } 49 ; 59 ; 07 ; 0 \\
\text { E } 14 ; 45 ; 59 ; 7\end{array}$ & 412 & 10 & 18.5 & 511 & 8.17 & 20 & 2.0 & 0.37 & 1.85 & 7.194 & 45.59 \\
\hline L42 & Jevanský & $\begin{array}{l}\text { N } 49 ; 58 ; 43 ; 7 \\
\text { E } 14 ; 47 ; 13 ; 8\end{array}$ & 395 & 70 & 18.6 & 431 & 8.50 & 25 & 2.2 & 0.41 & 1.39 & 4.664 & 59.79 \\
\hline L43 & Pařez & $\begin{array}{l}\text { N } 49 ; 59 ; 05 ; 5 \\
\text { E } 14 ; 46 ; 33 ; 5\end{array}$ & 418 & 70 & 18.4 & 534 & 7.39 & 25 & 2.5 & 0.34 & 1.51 & 7.159 & 19.30 \\
\hline L44 & Líšnice & $\begin{array}{l}\text { N } 49 ; 45 ; 42 ; 0 \\
\text { E } 16 ; 51 ; 39 ; 0\end{array}$ & 320 & 0 & 18.0 & 457 & 7.56 & 50 & 1.7 & 0.19 & 1.15 & 5.635 & 45.82 \\
\hline L45 & Obectov & $\begin{array}{l}\text { N } 49 ; 43 ; 39 ; 0 \\
\text { E } 16 ; 55 ; 43 ; 0\end{array}$ & 329 & 100 & 18.0 & 296 & 7.26 & 65 & 3.7 & 0.29 & 0.66 & 4.833 & 185.06 \\
\hline
\end{tabular}


The genera Geitlerinema, Komvophoron, Phormidium and Pseudanabaena occurred in the majority of samples. The most frequent species occurring in high abundances were the following: $P$ s. catenata, Ph. tergestinum, $K$. minutum, $K$. constrictum and G. splendidum. Geitlerinema was lacking or rare in the localities with high conductivity $\left(>500 \mu \mathrm{S} . \mathrm{cm}^{-1}\right)$. Cyanobacterial species new for the Czech Republic or science within the genera Komvophoron and Isocystis will be discussed elsewhere (HAŠLER \& PoulíčKová, unpublished). All taxa occurring under anoxic conditions were motile, poorly pigmented/almost colourless filamentous forms (Arthrospira, Komvophoron, Phormidium, Spirulina). Planktic/ meroplanktic forms were represented by genera Aphanocapsa, Merismopedia, Microcystis, Planktothrix, dominating usually at the same time in pelagial.

\section{Occurrence of desmids}

In the course of the investigation, 42 desmid taxa were encountered in epipelon-samples (Table 2, Figs 17-27). Most of them belong to commonly occuring species with a wide ecological amplitude in relation to trophy and $\mathrm{pH}$. It is especially true of Closterium acerosum, $\mathrm{Cl}$. acutum, $\mathrm{Cl}$. moniliferum, Cl. tumidulum, Cl. venus, Cosmarium formosulum, C. laeve, C. reniforme, Staurastrum tetracerum and Staurodesmus cuspidatus occupying various types of aquatic habitats from (moderately) acidic, (oligo-)mesotrophic to alkaline, eutrophic ones (RŮŽıčKA 1977, COESEL 1998, Lenzenweger 1997, 1999, 2003). These species were distributed within all site groups (No. I-IV., Fig. 2). Moreover, Closterium acerosum, Cl. leibleinii, $\mathrm{Cl}$. moniliferum and $\mathrm{Cl}$. tumidulum rank among the few desmids able to endure higher levels of eutrophication and saprobity (RŮŽǏ̌KA 1977, Coesel 1983, Lenzenweger 1996). High abundances of such species are indicative of water eutrophication (GuTOwsKI et al. 2004). From this species group. $\mathrm{Cl}$. acerosum occured mostly at sediments with high proportion of organic material (group No. II).

Besides these common species, several remarkable taxa were found mostly at oligo/ dystrophic sites (group No. III). Closterium pseudolunula seems to be rather rare in middle Europe (RŮŽIČKA 1977, LENZENWEGER 2003) and only 3 records have been published from the Czech Republic (PoulíčKová et al. 2004b). Cosmarium humile, widely distributed especially in mesotrophic, moderately acid to alkaline aquatic sites, is indicative of mature, relatively stable ecosystems (Coesel 1991, 1998). C. laeve var. octangulare, araretaxoninhabitingmainlymediumslightly acid, mesotrophic waters (LENZENWEGER 2003) and C. laeve var. pseudooctangulare rarely found in slightly eutrophic, neutral-alkline water bodies (COESEL 1998, LENZENWEGER 2003) are new for the Czech Republic (PoulíčKoví et al. 2004b). C. subprotumidum, evaluated as rare by LENZENWEGER (2003), is reported for the first time from Moravia (PoulíčKovÁ et al. 2004b). Coesel (1998) assigned it indicative of mature ecosystems. C. variolatum var. cataractarum, an acidophilous, mesotrophic alga, rare in Europe (Coesel 1998, Lenzenweger 2003) and highly indicative of finely balanced, mature ecosystems (Coesel 1998) may be considered as a new taxon for the Czech Republic (PoulíčKovÁ et al. 2004b). The species $C$. vexatum var. lacustre, a rare taxon in central Europe (Lenzenweger 2003), has not been recorded in our country so far (PoulíčKovÁ et al. 2004b). C. turpinii var. podolicum is a conspicuous desmid with tendency to occure in stable ecosystems of mesotrophic, neutral-alkline character (COESEL 1998). It has been sparcely reported from the territory of the Czech Republic (e.g. FISHER 1920, RÚŽIČKA 1957).

Staurastrum alternans, found at Vavrouškův pond (group No. III), may serve as an indicator of stable ecosystems (CoEsel 1998). St. blocklandiae (new for the Czech Republic), prefering neutralalkline, slightly eutrophic waters, is considered to be very rare by LENZENWEGER (2003), but it may be more frequent (COESEL 1997). It could be previously over-looked or is spreading recently. St. brachiatum (new for Moravia region) is characterized as an acidophilous, oligomesotrophic species indicating mature ecosystems (Coesel 1998, Lenzenweger 2003). Although, the vaste majority of desmids live in association with substrate, they can be often observed in pelagial. The truly planktic (euplanktic) species living in large water bodies are rather exceptions among desmids (RŮžIČKa 1977, Coesel 1998). These lifeform preferences were clearly reflected in species assortment recorded during the study (Table 2).

\section{Occurrence of other algae/organisms}

The distribution of diatoms has been published elsewhere (LysÁKOVÁ et al. 2007). Euglenophytes were represented by genera Euglena, Phacus, Trachelomonas and several apochromatic taxa 
Table 2. List of cyanobacteria and desmids from the surface of bottom sediments. For localities see Table 1; living forms: E epipelic, L - litoral, $\mathrm{P}$ - planktonic species.

\begin{tabular}{|c|c|c|}
\hline Taxon & Locality & Form \\
\hline \multicolumn{3}{|l|}{ Cyanobacteria - coccal } \\
\hline Aphanocapsa sp. & $24,36,45$ & $\mathrm{~L}$ \\
\hline Chroococcus limneticus LeMMERM. & $2,7,18,19$ & $\mathrm{~L}$ \\
\hline Coelomoron pusillum (VAN GOOR) KOMÁREK & 25,38 & $\mathrm{~L}$ \\
\hline Cyanogranis bassifixa HINDÁK & 14,19 & $\mathrm{~L}$ \\
\hline Cyanogranis ferruginea (WAWRIK) HINDÁK & 45 & $\mathrm{~L}$ \\
\hline Merismopedia elegans A.BRAUn in KÜTZ. & $18,22,25,29,34,36,39,44$ & $\mathrm{~L}$ \\
\hline Merismopedia punctata MEYEN & $9,10,13,19,33,34,45$ & $\mathrm{~L}$ \\
\hline Microcystis aeruginosa (KüTz.) KüTz. & $24,25,37,42$ & $\mathrm{P}$ \\
\hline $\begin{array}{l}\text { Microcystis wesenbergii (КOMÁREK) КOMÁREK in } \\
\text { KONDRATEVA }\end{array}$ & 25 & $\mathrm{P}$ \\
\hline Snowella litoralis (HÄYRÉN) KOMÁREK et HINDÁK & $10,15,42,45$ & $\mathrm{~L}$ \\
\hline \multicolumn{3}{|l|}{ Cyanobacteria - filamentous } \\
\hline Anabaena sp. & 14 & $\mathrm{~L}$ \\
\hline Aphanizomenon gracile & 14 & $\mathrm{P}$ \\
\hline Arthrospira jenneri STIZENBERGER ex GOMONT & 45 & $\mathrm{E}$ \\
\hline Geitlerinema amphibium (AGARDH ex Gomont) ANAGN. & $2,4,6,5,22,23,26,29,39$ & $\mathrm{E}$ \\
\hline Geitlerinema splendidum (Grev. ex Gomont) AnaGn. & $3,6,9,10,11,12,13,14,16,17$ & $\mathrm{E}$ \\
\hline Isocystis cf. pallida Woron. & $18,36,43$ & $\mathrm{E}$ \\
\hline $\begin{array}{l}\text { Komvophoron constrictum (SZAFER) ANAGN. et KOMÁ- } \\
\text { REK }\end{array}$ & $2,7,13,14,18,22,24,38,39,42,45$ & $\mathrm{E}$ \\
\hline Komvophoron minutum (SKUJA) ANAGN. et KomÁreK & $\begin{array}{l}2,3,6,9,11,12,13,15,16,19,22,23,24,25,2 \\
8,2930,31,33,36,40,42,45\end{array}$ & $\mathrm{E}$ \\
\hline Komvophoron schmidlei (JAAG) ANAGN. et KoMÁREK & $1,9,10,13,15,38$ & $\mathrm{E}$ \\
\hline Komvophoron sp. & $22,25,36,40$ & $\mathrm{E}$ \\
\hline Komvophoron sp. & 9 & $\mathrm{E}$ \\
\hline Komvophoron sp. & 22 & $\mathrm{E}$ \\
\hline Limnothrix redekei (VAN GOOR) MEFFERT & 38 & $\mathrm{P}$ \\
\hline Nostoc sp. & 11 & $\mathrm{E}$ \\
\hline Oscillatoria limosa AgardH ex Gomont & $1,3,7,12,18,22,29,35$ & $\mathrm{E}$ \\
\hline $\begin{array}{l}\text { Phormidium acuminatum (GoMONT) ANAGN. et KomÁ- } \\
\text { REK }\end{array}$ & $19,29,33,34,42$ & $\mathrm{E}$ \\
\hline $\begin{array}{l}\text { Phormidium autumnale [AGARDH] TREvisAn ex Go- } \\
\text { MONT }\end{array}$ & $3,4,5,8,9,10,14,15,22,35,37,45$ & $\mathrm{E}$ \\
\hline $\begin{array}{l}\text { Phormidium chalybeum (MERTEns ex Gomont) AnaG. } \\
\text { et KOMÁreK }\end{array}$ & $7,18,22,24,38,43,45$ & $\mathrm{E}$ \\
\hline $\begin{array}{l}\text { Phormidium simplicissimum (GOMONT) ANAGN. et } \\
\text { KOMÁREK }\end{array}$ & 9,12 & $\mathrm{E}$ \\
\hline $\begin{array}{l}\text { Phormidium terebriforme (AGARDH ex GomONT) ANA- } \\
\text { GN. et KOMÁREK }\end{array}$ & $5,7,29,40,43,44$ & $\mathrm{E}$ \\
\hline Phormidum tergestinum [KÜTz.] ANAGn. et KoMÁREK & $\begin{array}{l}1,2,3,4,6,8,9,12,13,14,15,18,19,20,21,2 \\
2,29,33,34,41,43,44,45\end{array}$ & $\mathrm{E}$ \\
\hline Phormidium sp. & $19,36,38$ & $\mathrm{E}$ \\
\hline
\end{tabular}


Table 2 Cont.

\begin{tabular}{|c|c|c|}
\hline Taxon & Locality & Form \\
\hline Phormidium sp. & $21,22,32$ & $\mathrm{E}$ \\
\hline $\begin{array}{l}\text { Planktolyngbya limnetica (LEMMERM.) KOMÁRK.-LEGN. } \\
\text { et CRONBERG }\end{array}$ & 10 & $\mathrm{P}$ \\
\hline Planktothrix agardhii (GOMONT) ANAGN. et KOMÁREK & $9,13,37$ & $\mathrm{P}$ \\
\hline Pseudanabaena catenata LAUTERBORN & $\begin{array}{l}1,2,3,4,5,6,7,8,9,10,11,15,16,18,22,23 \\
24,25,26,2930,31,32,33,36,40,42,43,4 \\
4,45\end{array}$ & $\mathrm{E}$ \\
\hline Pseudanabaena galeata BöcheR & $12,13,14,15,17,28,43$ & $\mathrm{~L}$ \\
\hline Pseudanabaena limnetica (LEMMERM.) KOMÁREK & $7,9,13,14,15,22$ & $\mathrm{~L}$ \\
\hline Spirulina major KÜTz. ex GomonT & $2,18,19,22,40$ & $\mathrm{E}$ \\
\hline \multicolumn{3}{|l|}{ Zygnematophyceae - Desmidiales } \\
\hline Closterium cf. acerosum (SCHRANK) EHRENB. ex RALFS & $7,23,25,40,41,43$ & $\mathrm{E}$ \\
\hline Closterium acutum BRÉB. & 26,36 & $\mathrm{E}$ \\
\hline Closterium leibleinii KüTz. ex RALFS & 12,28 & $\mathrm{E}$ \\
\hline Closterium limneticum LEMMERM. & $17,27,37,39,42$ & $\mathrm{P}$ \\
\hline Closterium moniliferum (BORY) EHRENB. ex RALFS & 31 & $\mathrm{E}$ \\
\hline $\begin{array}{l}\text { Closterium praelongum var. brevius (NORDST.) WILLI } \\
\text { KRIEG. }\end{array}$ & 32 & $\mathrm{P}$ \\
\hline Closterium pseudolunula BORGE & $7,12,36$ & $\mathrm{E}$ \\
\hline Closterium cf. tumidulum GAY & 2,30 & $\mathrm{E}$ \\
\hline Closterium venus Kütz. ex RALFs & $29,30,31$ & $\mathrm{E}$ \\
\hline Closterium sp. & 24 & $\mathrm{E}$ \\
\hline Cosmarium biretum var. trigibberum NoRDST. & 2,12 & $\mathrm{E}$ \\
\hline Cosmarium botrytis cf. var. tumidum Wolle & 2,29 & $\mathrm{E}$ \\
\hline Cosmarium formosulum HofF. in Nordst. & 33 & $\mathrm{E}$ \\
\hline Cosmarium granatum BRÉB. in RALFS & $2,12,21,22,31,34$ & $\mathrm{E}$ \\
\hline Cosmarium humile (GAY) Nordst. in DE TONI & 31 & $\mathrm{E}$ \\
\hline Cosmarium impressulum ELFVING & 26,31 & $\mathrm{E}$ \\
\hline Cosmarium laeve RABENH. & $2,22,25,40$ & $\mathrm{E}$ \\
\hline $\begin{array}{l}\text { Cosmarium laeve var. octangulare (WILLE) WEST et G. } \\
\text { S. WEST }\end{array}$ & 29 & $\mathrm{E}$ \\
\hline $\begin{array}{l}\text { Cosmarium laeve var. pseudooctangulare FRITSCH et } \\
\text { RICH }\end{array}$ & 12,24 & $\mathrm{P}$ \\
\hline Cosmarium cf. praecisum BORGE & 31 & $\mathrm{E}$ \\
\hline Cosmarium pseudoornatum EICHLER et GUTw. & 32 & $\mathrm{E}$ \\
\hline Cosmarium regnellii $\mathrm{W}$ ILLE & 2,31 & $\mathrm{E}$ \\
\hline Cosmarium reniforme (RALFS) ARCHER & 29 & $\mathrm{E}$ \\
\hline Cosmarium subcrenatum HANTZSCH & 12 & $\mathrm{E}$ \\
\hline Cosmarium subgranatum (NORDST.) LÜTKEM. & 30 & $\mathrm{E}$ \\
\hline Cosmarium subprotumidum NoRDST. & 22 & $\mathrm{E}$ \\
\hline Cosmarium tenue ARCHER & 26 & $\mathrm{E}$ \\
\hline Cosmarium turpinii var. podolicum GuTw. & 33 & $\mathrm{E}$ \\
\hline Cosmarium variolatum var. cataractarum RACIB. & 31,32 & $\mathrm{E}$ \\
\hline Cosmarium vexatum var. lacustre MEssiK. & $22,30,33$ & $\mathrm{E}$ \\
\hline
\end{tabular}


Table 2 Cont.

\begin{tabular}{llc}
\hline Taxon & Locality & Form \\
\hline Euastrum insulare (WITTR.) RoY & 31 & $\mathrm{E}$ \\
Penium margaritaceum (EHRENB.) ex BRÉB. & 2 & $\mathrm{E}$ \\
Staurastrum alternans (BRÉB.) RALFS & 29 & $\mathrm{E}$ \\
Staurastrum anatinum f. paradoxum (MEYEN) BROOK & 27 & $\mathrm{E}$ \\
Staurastrum blocklandiae COESEL et JOOSTEN & 37,42 & $\mathrm{P}$ \\
Staurastrum brachiatum RALFS & 20,23 & $\mathrm{E}$ \\
Staurastrum cf. manfeldtii DeLPONTE & 26 & $\mathrm{E}$ \\
Staurastrum cf. planctonicum TEILING & 27,42 & $\mathrm{P}$ \\
Staurastrum tetracerum (KÜTZ.) RALFS & $24,31,37,42$ & $\mathrm{P}$ \\
Staurastrum sp. & 29 & $\mathrm{E}$ \\
Staurodesmus cuspidatus (BRÉB. ex RALFS) TEILING & 31 & $\mathrm{E}$ \\
Xanthidium antilopaeum (BRÉB.) KÜTZ. & 31 & $\mathrm{E}$ \\
\hline
\end{tabular}

(e.g. Anisonema, Entosiphon). The highest species richness was found on the muddy sediments (group No. II.) or anoxic muddy sediments (group No. IV.). The most frequent species were: Euglena spirogyra EhrenB., E. texta (Dujard.) Lemmerem., Phacus caudatus HüBNER, P. longicauda (EHRENB.) Dujard., $P$. monilatus Stokes, $P$. orbicularis HüBNER, Strombomonas eurystoma Popova, Trachelomonas hispida (PERTY) SteIN. The sandy bottom sediments (group No. III.) were poorly colonised, e.g. E. acus. Other epipelic organisms were represented by Gymnodinium aeruginosum F.STEIn, Paulinella chromatophora LAUTERBORN and various protozoan, feeding on epipelic algae (Urceolus cyclostomus (F.STEIN) MERESCHK., Amoeba).

\section{Discussion}

Epipelic assemblages at 45 ponds covering trophic and $\mathrm{pH} /$ conductivity gradients were found highly diverse, even more than expected. They were dominated by diatoms (185 species; Poulíčková, unpublished.), cyanobacteria (39 species) and desmids (42 species). Other groups were less abundant. Round (1953) have found in Malhalm Tarn 16 species of cyanobacteria (Coelosphaerium kuetzingianum NäGELI, C. pusillum VAN GoOR, Aphanothece stagnina (SPreng.) A.Braun in RaBenh., Chroococcus turgidus (KÜTz.) NäGELI, Synechococcus aeruginosus NäGELI, Merismopedia glauca (ЕHRENB.) Kütz., Pseudanabaena catenata LAUTERBorn, Anabaena constricta (SzAFer) GeItLer, Oscillatoria limosa
Agardh ex Gomont, O. splendida Grev. ex Gomont, O. irrigua Kütz. ex Gomont, Microcystis spp. and Cylidrospermum spp.) and 9 species of desmids (Netrium oblongum (DE BARY) LÜTKEM., N. digitus (Ehrenb.) Itzigs. et Rothe, Cosmarium punctulatulum BRÉB., Closterium ehrenbergii Menegh. ex Ralfs, C. moniliferum (Bory) Ehrenb. ex Ralfs, C. aciculare T. West, Euastrum pectinatum (BRÉB.) BRÉB., E. denticulatum GAY). Although, data recorded by Round (1957) from English Lake District (cyanobacteria 20 species, desmids 35 species) are comparable to ours, only few species can be considered as truly epipelic. That is a question, whether planktonic species, can be included into evaluation of the epipelon. We tried to highlight dominant lifestyle of species (based on our experience and published data) in Table 2.

The distribution of epipelic species (cyanobacteria and desmids) seems to be influenced primarily by sediment quality. Statistic evaluation based on all environmental data divided localities into 4 groups with different sediment quality. Group I. can be characterized by sandymuddy sediments and low conductivity, group II. had high proportion of organic detritus and high conductivity, group III. include oligo/dystrophic sites with sandy sediments and group IV. eutrophic localities with muddy sediments.

Round (1953) recognized four basic types of sediments, which were found in Malhalm Tarn (UK) - black flocculent peat nature, containing the partially decayed fibrous remain of Angiosperms and Bryophytes; greyish calcareous sediment, containing sand grains; sediment with encrusted 
Chara fragments; none calcium carbonate peat sediments with fragments of higher plants. These cannot fully correspond with our types, because of geological uniqueness of Malhalm Tarn region. Lakes within English Lake District were divided (Round 1957) only into two groups according to amount of organic content: 1) more productive lakes dominated by cyanobacteria with organic sediments or higher amount of organic substances in the sediments (above 26\%), 2) the "rocky“ lakes with very low cyanobacterial produktivity and with an organic content below $22 \%$.

The similar pattern can also be found in Czech ponds, but the productivity of fishponds is mostly artificial, influenced by human impacts and the type of management (PoulíčKové et al. 2008). Eutrophic localities used for intensive fish production had high proportion of euglenophytes and cyanobacteria. At the same time, no desmids or just few desmid species tolerant to such conditions were recorded there. On the contrary, nutrient poor water bodies with sandy sediments (group III.) could be characterised by occurrence of remarkable desmids. Our data could not bring any information about the seasonal changes within the epipelic assemblages, studied previously by Round (1961). The seasonal maximum of epipelon can be expected from March - June, while cold months (October - February) can be considered as seasonal minimum in English Lake District area (Round 1961).

Lakes/ponds accumulate vast amounts of ecological and chemical information in their sediments, which can be used to reconstruct past changes in lake ecosystems (HALL \& SMOL 2001, PoulíčKová et al. 2008c). As speciesspecific responses to environmental changes occur, particularly diatoms/desmids are useful bioindicators in the study of anthropogenically mediated environmental change (COESEL 1998, 2003, Hall \& Smol 2001). However, the use of epipelon for biomonitoring of recent environmental changes has caused controversy. Epilithon is considered as the most suitable substrate for monitoring streams (Round 1991), but sampling these substrates in lakes may be problematic due to their sporadic occurrence. Although stones are available in mountain lakes, they could be missing in other still-water systems. Some authors have reported lower correlations between the epilithon and environmental factors in comparison to epipelon or epipsammon (PASSY et al. 1999). We did not find any significant correlation between epipelic assemblages and environmental variables measured in water column (Canonical Correspondence Analysis, not illustrated). Analysis of different substrates may yield variable results with variation being dependent on the environmental conditions at the time of sampling. For example, in a recent study of a perialpine lake, the diatom assemblage on reed stalks yielded an underestimated trophic status whereas consideration of the epipelon resulted in an overestimation (PoulíčKovÁ et al. 2004a). Differences in substrate-specific estimates were not, however, significant in the case of eutrophic lowland ponds (KitNer \& PoulíčKovÁ 2003). Potapova \& Charles (2005) concluded that the choice of substrate to be sampled should depend on the assessment indicators to be used. If the indicators are based on the autecologies of many algal taxa (e.g. inference models or autecological indices), there is little requirement for substrate restrictions. However, it should be noted that many sediment samples contain a high proportion of planktonic species (PoulíčKovÁ et al. 2004a) making taxonomic separation of many epipelic diatoms (species complexes) problematic. The ecological requirements of many epipelic species are not sufficiently known and there is little critical evidence to support the epipelon as suitable ecological indicators in one way or another (SCHÖNFELDER et al. 2002, KING et al. 2006). Moreover, cyanobacteria are extremely phenotypically polymorphic, which complicate their identification. On the other hand, GutowsKi et al. (2004) distinguished 74 benthic algal species as useful indicators for the assement of ecological status of siliceous aquatic sites in highlands of Germany. Regarding the discussed problems, desmids appear to be suitable enough for biomonitoring purposes as their ecological preferences are well known and the association with substrate is less tight compared to many diatoms and cyanophytes. So, if they occupy the pelagial environment as well, their occurrence in particular water bodies is determined by both physico-chemical variables of water and sediment. Finally, it can be expected that the desmid related monitoring results are not substrate dependent.

\section{Acknowledgement}

This research was supported by project GACR 206/07/0115 from the Grant agency of the Czech Republic. 


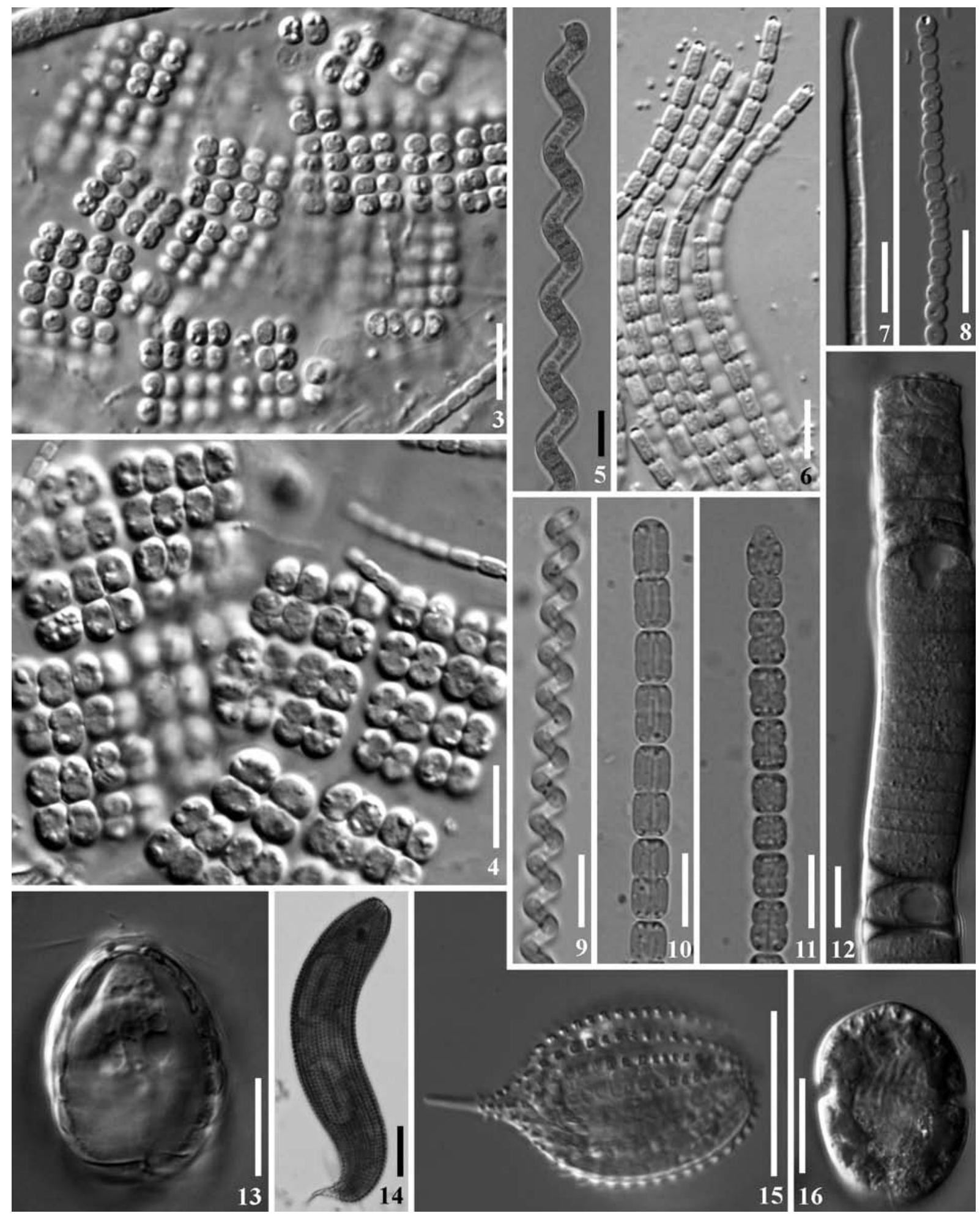

Figs 3-16. Representatives of epipelic cyanobacteria and eukaryotic flagellates: 3 - Merismopedia punctata; 4 - M. elegans; 5 - Arthrospira jenneri; 6 - Pseudanabaena catenata; 7 - Geitlerinema splendidum; 8 - Isocystis cf. pallida; 9 - Spirulina major; 10,11-Komvophoron constrictum; 12 - Oscillatoria limosa, hormogonia formation; 13 - Paulinella chromatophora; 14 - Euglena spirogyra; 15 - Phacus monilatus var. suecicus; 16 - Gymnodinium aeruginosum. 

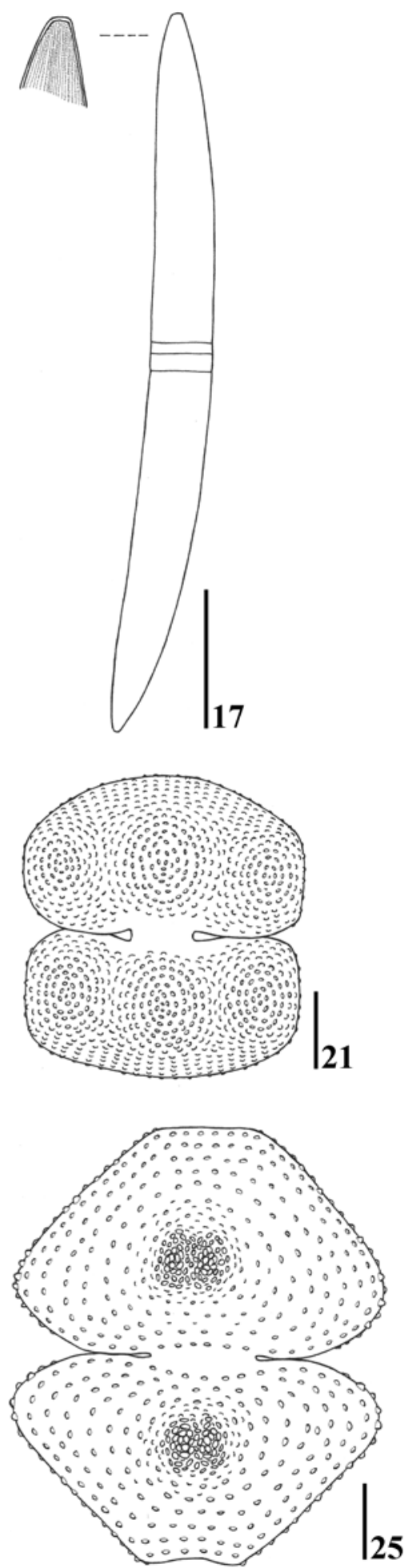
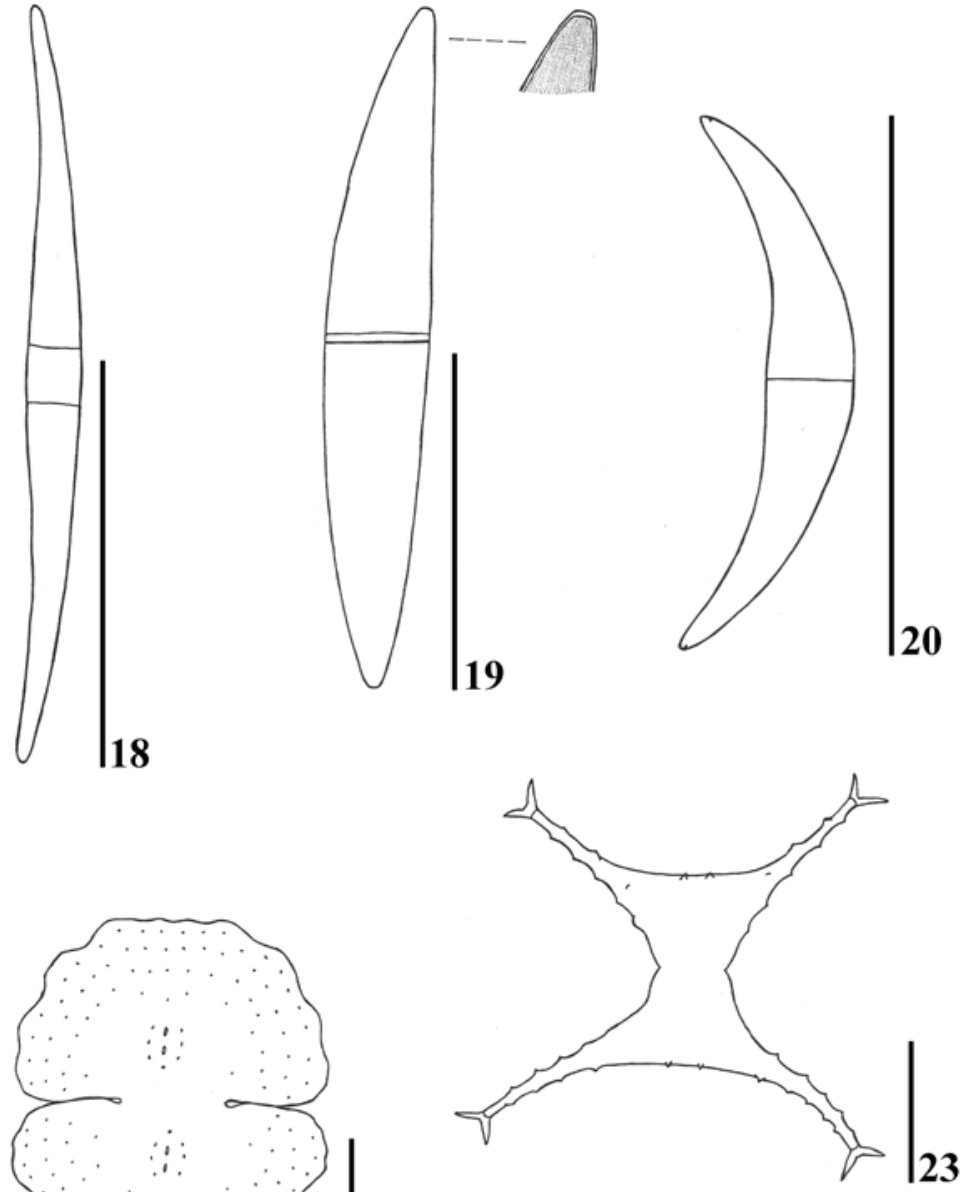

22
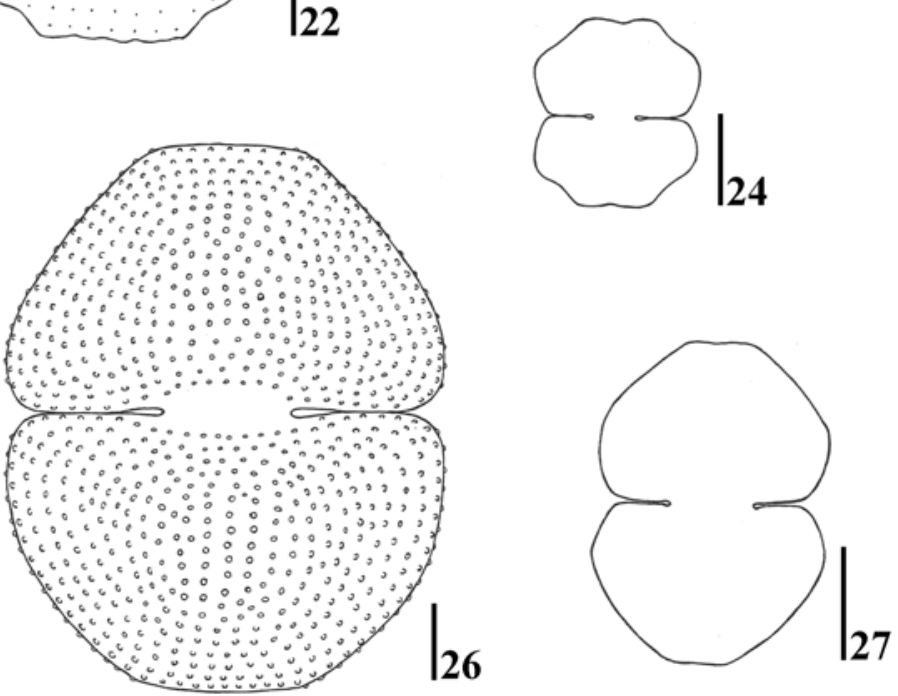

Figs 17-27. Representatives of epipelic desmids: 17 - Closterium cf. acerosum; 18 - Closterium praelongum var. brevius; 19 - Closterium pseudolunula; 20 - Closterium cf. tumidulum; 21 - Cosmarium biretum var. trigibberum; 22 - Cosmarium subprotumidum; 23 - Staurastrum blocklandiae; 24 - Cosmarium laeve var. pseudooctangulare; 25 - Cosmarium turpinii var. podolicum; 26 -Cosmarium botrytis $\mathrm{cf}$. var. tumidum; 27 - Cosmarium laeve. 


\section{References}

Belmonte, G. A., Rubino, M. F. \& Boero, F. (1997): Morphological convergence of resting stages of planktonic organisms: a review. - Hydrobiologia 355: 159-165.

Bold, H. C. (1949): The morphology of Chlamydomonas chlamydogama sp. nov. - Bull. Torrey Bot. Club. 76: 101-108.

BURKHOLDER, J. M. (1996): Interactions of benthic algae with substrata. - In: Stevenson, R.J., Bothwell, M.L. \& Lowe, R.L. (ed): Algal ecology: freshwater benthic ecosystems, Sec. 2 Factors affecting benthic algae, 253-297 pp., Academic press, San Diego.

Coesel, P. F. M. (1983): De Desmidiaceeën Van Nederland - Sieralgen. Deel 2 Fam. Closteriaceae. - 50 pp., Wetenschappelijke Mededelingen K. N. N. V., Hoogwoud NH.

Coesel, P. F. M. (1991): De Desmidiaceeën Van Nederland. Deel 4 Fam. Desmidiaceae (2). - 89 pp., Stichting Uitgeverij K. N. N. V., Utrecht.

Coesel, P. F. M. (1997): De Desmidiaceeën Van Nederland. Deel 6 Fam. Desmidiaceae (4). - 93 pp., Stichting Uitgeverij K. N. N. V., Utrecht.

Coesel, P. F. M. (1998): Sieralgen en Natuurwaarden. - 57 pp., Stichting Uitgeverij K. N. N. V., Utrecht.

Coesel, P. F. M. (2003): Desmid flora data as a tool in conservation management of Dutch freshwater wetlands. - Biologia 58: 717-722.

Fisher, R. (1920): Die Algen Mährens und ihre Verbreitung.Verh. D. Nat. Vereins in Brünn 57: 1-94.

Gutowski, A., Foerester, J. \& Schaumburg, J. (2004): The use of benthic algae, excluding diatoms and charales, for the assesment of the ecological status of running fresh waters: a case history from Germany. - Oceanological and Hydrobiological Studies 33(2): 3-15.

Hall, R. I., Smol J. P. (2001): Diatoms as indicators of lake eutrophication. - In: Stoermer, E. F., Smol, J. P. (ed): The Diatoms: Applications for the Environmental and Earth Sciences, 469 pp. Cambridge Univ. Press.

Happey-Wood, C. M. (1988): Vertical-migration patterns of flagellates in a community of freshwater benthic algae. - Hydrobiologia 161: 99-123.

HeKera, P. (1999): Vliv antropogenní činnosti na chemismus reky Moravy [The human impacts to chemistry of the Morava River]. - 82 pp., PhD thesis, Masaryk University, Brno.

King, L., Glarke, G., Bennion, H., Kelly, M. \& Yallop, M. (2006): Recommendations for sampling littoral diatoms in lakes for ecological status assessments. J. Appl. Phycol. 18: 15-25.

Kitner M. \& PoulíčKová A. (2003): Littoral diatoms as indicators for the eutrophication of shallow lakes. Hydrobiologia 506-509: 519-524.

LENZENwEGER, R. (1996): Desmidiaceenflora von Österreich. - In: Crammer, J. (ed.): Bibliotheca Phycologica 101/1: 1-162 pp., Gebrüder Borntraeger Verlagsbuchhandlung, Berlin- Stuttgart.

LENZENwEgER, R. (1997): Desmidiaceenflora von Österreich. - In: Crammer, J. (ed.): Bibliotheca Phycologica 102/2: 1-216 pp., Gebrüder Borntraeger Verlagsbuchhandlung, Berlin- Stuttgart.

LenZenweger, R. (1999): Desmidiaceenflora von Österreich. In: Crammer, J. (ed.): Bibliotheca Phycologica 104/3:
1-218 pp., Gebrüder Borntraeger Verlagsbuchhandlung, Berlin- Stuttgart.

Lenzenweger, R. (2003): Desmidiaceenflora von Österreich. - In: Crammer, J. (ed.): Bibliotheca Phycologica 111/4: 1-87 pp., Gebrüder Borntraeger Verlagsbuchhandlung, Berlin- Stuttgart.

Lock, M. A., Wallace, R. R., Costerton, J. W., Ventullo, R. M. \& CHARLton, S. E. (1984): River epiliton: Toward a structural-functional model. - Oikos 42: 10-22.

LysákovÁ, M., Kitner, M. \& PoulíčKovÁ, A.(2007): The epipelic algae at fishponds of Central and Northern Moravia (The Czech Republic). - Fottea 7: 69-75.

Mann, D. G. (1999): The species concept in diatoms. Phycologia 38: 437-495.

ManN, D. G. \& Droop, S. J. M. (1996): Biodiversity, biogeography and conservation of diatoms. Hydrobiologia 336: 19-32.

Mann, D. G., Chepurnov, V. A. \& Droop, S. J. M. (1999): Sexuality, incompatibility, size variation, and preferential polyandry in natural populations and clones of Sellaphora pupula (Bacillariophyceae). J. Phycol. 35: 152-170

Mann, D. G., Thomas, S. J. \& Evans, K. M. (2008): Revision of the diatom genus Sellaphora: a first account of the larger species in the British Isles. - Fottea 8: 15-78.

Paerl, H. W. (1990): Physiological ecology and regulation of N2 fixation in natural waters. - In: MARShaLL, K. C. (ed.): Advances in microbial ecology 2: 261-315 pp., Plenum, New York.

Passy, S. I., Pan ,Y. Lowe, R. L. (1999): Ecology of the major periphytic diatom communities from the Mesta River, Bulgaria. - Int. Rev. Ges. Hydrobiol. 84: 129-174.

Potapova, M. R. \& Charles, D. F. (2002): Benthic diatoms in USA rivers: distributions along spatial and environmental gradients. - J. Biogeogr. 29: 167187.

PoulíčKová, A., Duchoslav, M. \& Dokulil, M. (2004a): Littoral diatom assemblages as bioindicators for lake trophy: A case study from alpine and pre-alpine lakes in Austria. - Eur. J. Phycol. 39(2): 143-152.

PoulíčKová, A., Lhotský, O. \& Dř́malová, D. (2004b): Prodromus sinic a řas ČR. - Czech Phycology 4: 19-33.

Poulíčková, A., Hašler, P., Lysáková, M. \& Spears, B. (2008a): The ecology of freshwater epipelic algae: an update. - Phycologia 47(5), in press.

PoulíčKová, A., Š́pačKová, J., Kelly, M. G., Duchoslav, M. \& MANN, D. G. (2008b): Ecological variation within Sellaphora species complexes (Bacillariophyceae): specialists or generalists? - Hydrobiologia, in press.

PoulíčKová, A., Lysáková, M., Hašler, P. \& Lelková, E. (2008c): Fishpond sediments - the source of palaeoecological information and algal ,seed banks“. - Nova Hedwigia 86: 141-153.

PoulíčKovÁ, A. \& ManN, D. G. (2008): Autogamous auxosporulation in Pinnularia nodosa (Bacillariophyceae). - J. Phycol. 44: in press.

Round, F. E. (1953): An investigation of two benthic algal communities in Malhalm tarn, Yorkshire. - J. Ecol. 174-197.

Round. F. E. (1957): Studies on bottom-living algae in some lakes of the English lake district: part III. The distribution on the sediments of algal groups other than the Bacillariophyceae. - J. Ecol. 45(3): 649- 
664.

Round, F. E. (1961): Studies on bottom-living algae in some lakes of the English lake district, part V. The seasonal cycles of the Cyanophyceae. - J.Ecol. 49(1): 31-38.

Round, F. E. (1972): Patterns of seasonal succession of freshwater epipelic algae. - Br.phycol. J. 7: 213220.

Round, F. E. (1991): Use of diatoms for monitoring rivers. - In: Whitton, B. A., Rott, E. Friedrich, G. (eds): Use of algae for monitoring rivers, Proceedings of an International Symposium, 25-33 pp., Duseldorf, Germany.

Round, F. E. \& EAton, J. W. (1966): Persistent, verticalmigration rhythms in benthic microflora: III. The rhythm of epipelic algae in a freshwater pond. $-\mathrm{J}$. Ecol. 54: 609-615.

RŮŽIČKA, J. (1957): Krásivky horní Vltavy (Šumava). Preslia 29: 132-154.

RŮŽIČKA, J. (1977): Die Desmidiaceen Mitteleuropas. - 291 pp., Lieferung. E. Schweizerbart'sche Verlagsbuchhandlung, Stuttgart.
Schönfelder, I., Gelbrecht, J., Schönfelder, J. \& Steinberg, Ch. E. W. (2002): Relationships between littoral diatoms and their chemical environment in northeastern german lakes and rivers. - J. Phycol. 38: 66-82.

Sicko-Goad, L., Stoermer, E. F. \& Kociolek, J. P. (1989): Diatom resting cell rejuvenation and formation: Time course, species records, and distribution. - Journal of Plankton Research 11: 375-389.

STAUB, R. (1961): Ernährungsphysiologisch-autökologische Untersuchungen an der planktonischen Blaualge Oscillatoria rubescens DC. - Schweiz. Z. Hydrol. 23: 82-198.

VERNON, L. P. (1960): Spectrophotometric determination of chlorophylls and pheophytins in plant extracts. Anal. Chem. 32: 1144-1150.

(C) Czech Phycological Society

Received January 8, 2008

Accepted May 20, 2008 